\title{
Ensemble Classifier based Approach for Classification of Examination Questions into Bloom's Taxonomy Cognitive Levels
}

\author{
K. A. Osadi \\ University of Colombo School of Computing \\ Colombo, Sri Lanka
}

\author{
M. G. N. A. S. Fernando \\ University of Colombo School of Computing \\ Colombo, Sri Lanka
}

\author{
W. V. Welgama \\ University of Colombo School of Computing \\ Colombo, Sri Lanka
}

\begin{abstract}
The concept of Bloom's taxonomy cognitive domain has been broadly used as a guideline in preparing a reasonable examination paper that consists of questions belonging to various cognitive levels which are helpful in evaluating different capabilities of students. Currently, academicians identify Bloom's taxonomy cognitive level manually, but that is a tedious and a time-consuming task. Therefore, the use of automatic classification technique based on Bloom's taxonomy cognitive levels is highly needed. Several studies have been carried out to fulfill this task, but most of these studies have failed to address the overlapping keyword problem among Bloom's taxonomy cognitive levels, and most of these studies have not considered the semantic structure of the examination questions. To overcome these problems, this study proposes a question classification model using an ensemble classifier approach by combining four different classifiers; namely rule based, support vector machine, $\mathrm{k}$-nearest neighbor and Naive Bayes.The results of four different classifiers are integrated to derive the final corresponding Bloom's taxonomy cognitive level, using majority voting and WordNet similarity values. WordNet similarity is used to explore the semantic structure of the examination questions. A sample of first year programming examination questions of University of Colombo School of Computing was used for the evaluation. Four domain experts confirmed identified Bloom's taxonomy cognitive levels of the questions in the dataset. The experimental results indicate that the proposed ensemble classifier approach yields much better accuracy than the accuracy of the individual classifiers.
\end{abstract}

\section{General Terms}

Ensemble Classifier, Bloom's Taxonomy, Rule Based, Machine Learning

\section{Keywords}

Bloom's taxonomy, Ensemble Classifier, k-nearest neighbor, Naive Bayes, Natural Language Processing, Rule based, Support Vector Machine

\section{INTRODUCTION}

knowledge, as an outcome of education, is no longer sufficient for fulfilling the requirements of the global information economy. Further, researchers have recently found that people who think critically and creatively are essential to the growth of any society in several aspects including economic, cultural, political, and psychological [4]. As a result, most educational organizations globally, have given more attention to developing the higher order thinking level skills of students and improving their learning achievements. Written examinations are one of the most famous and well-known methods for measuring student learning achievement and most educational organizations around the world use this technique to evaluate the achievement of the learning outcomes by their students [2]. As such, the examination questions in written examination papers, play a significant role in order to evaluate student knowledge and understanding of the particular subject. Hence, it is highly essential to prepare examination questions that assess different cognitive levels of students to develop both lower order and higher order thinking levels of students. However, creating such questions is very challenging for teachers and lecturers. In recent years, several educationists have developed different taxonomies which are a help to assess the different cognitive levels in examination questions. Among them, Bloom?s taxonomy cognitive domain has become a common reference in producing of examination questions [2].

There are three domains in the Bloom's taxonomy, namely; cognitive, affective and psychomotor. Bloom's taxonomy cognitive domain is more popular than other educational taxonomies due to its simple and well-structured levels and the fact that it can also be applied to a wide range of subjects [7]. Bloom's taxonomy cognitive domain has six levels as knowledge, comprehension, application, analysis, synthesis and evaluation. The first three levels are considered lower order cognitive levels while the remaining are considered higher order cognitive levels [7]. In 2001, Lorin Anderson and a group of educational specialists updated the original Bloom's taxonomy and this revised taxonomy is known as the revised Bloom's taxonomy. In revised Bloom's taxonomy, 


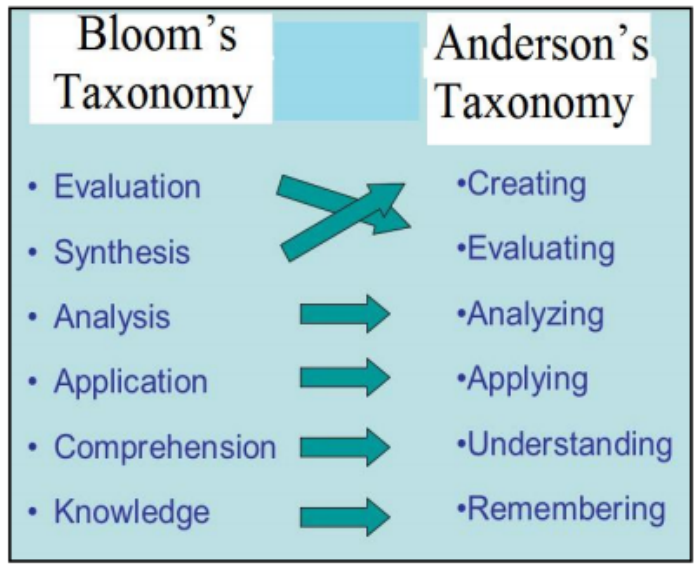

Fig. 1. Changes of original and revised Bloom's taxonomy cognitive levels

verbs are used to name cognitive categories instead of nouns. And also, three of the cognitive levels are renamed and top two higher orders cognitive levels are interchanged on original Bloom's taxonomy cognitive levels. The Revised Bloom's Taxonomy cognitive domain has six levels, namely; remember, under-stand, apply, analyze, evaluate and create. Fig. 1 shows the changes of original and revised Bloom's taxonomy. This study focuses on the levels of cognitive domain of the revised Bloom's taxonomy as it is closely related to student's knowledge and understanding of course materials.

In order to prepare balanced examination papers which means it includes both higher order and lower order cognitive levels questions and that cover defined learning objectives, lecturers need to identify, which questions belong to higher order cognitive levels and which questions belong to lower order cognitive levels. However, classifying examination questions to each Bloom's taxonomy cognitive levels manually by taking each examination question one by one is a difficult task as an examination questions may belong to more than one level of Bloom's taxonomy cognitive domain and in addition, the task itself is inherently time-consuming [2]. The difficulty of classifying examination questions into Bloom's taxonomy cognitive level manually gives rise to the need to utilize automatic classification techniques.

Several studies have been carried out to fulfill this task, however, most of these studies use a single classifier such as rule based approaches, Support Vector Machines (SVM), Naive Bayes (NB), k-Nearest Neighbor (KNN), artificial neural networks, statistical methods, etc but the problem is such a single classifier method cannot overcome the inherent drawbacks in each individual classifier. Statistical techniques (TF-IDF, C-Value, and N-gram) are not very suitable for question classification because these techniques require a large amount of data to obtain acceptable accuracy [7]. However, it is difficult to gather a large examination question dataset because these studies are often restricted to a specific subject domain. On the other-hand rule based approaches are tedious and time-consuming. With respect to portability and robustness, rule based systems have very low performance compared to other techniques [1]. Over the recent years, in an effort to overcome some of these difficulties, machine learning approaches have been formulated but these techniques also require a large amount of data for high accuracy and sometime, these techniques are memory intensive and hard to interpret.

Further, most of these studies have not considered the semantic structure of examination questions instead, have simply considered mapping keywords listed under each level of Bloom's taxonomy cognitive domain. Also, most of these studies fail to address the issue of overlapping keywords in Bloom's taxonomy cognitive levels. All the above reasons cause reduction of accuracy of the classification of previous studies. Further, recent researchers in the text classification field claim that the use of classifier combinations known as ensemble classifier approach, will improve classification results by bringing the advantages of each classifier and overcome their problems [11]. These are the facts that provide the motivation for this study.

Therefore, this study attempts to apply a classifier ensemble approach for the classification based on the hypothesis [10] that classifier ensembles are based on the reasoning that strengths and weaknesses of various classifiers can compensate each other. It is expected that this approach can solve the issue of the overlapping keyword problem of the Bloom's taxonomy cognitive levels and also increase the performance of classification since an instance which is misclassified by one classifier may be classified correctly by another classifier, thus pushing up the combined classification performance at the same time solving the overlapping keyword problem. Combining diverse classifiers is an essential characteristic for a successful classification [11]. Also in the combination phase, this study considers the semantic similarity of words and it also solves the problem of not considering the semantic similarity of examination questions, which was drawback found in past research studies.

\section{RELATED WORKS}

The task of the question classification is assigning a correct class label, depending on classification strategy, to a given question written in natural language. There were three main classification approaches identified such as rule based, supervised learning and classifier combination approaches for classifying examination questions into Bloom's taxonomy cognitive levels.

Rule based approaches try to match the questions with some manually handcrafted rules. These approaches, however, suffer from the need to define too many rules. Furthermore, while rule-based approaches may perform well on a particular dataset, they may have quite a poor performance on a new dataset and consequently it is difficult to scale them [1]. There were several studies carried out to classify examination questions into Bloom's taxonomy cognitive levels using rule based approach.

Syahidah Sufi Haris et al [1] proposed a rule based classification model to classify examination questions of computer programming subject. After applying NLP preprocessing techniques, they extracted POS tag patterns for each cognitive level and define those patterns as rules for each Bloom's taxonomy cognitive level. Syahidah Sufi Haris et al [8] enhanced above mention study [1] with n-gram statistical approach. If questions are not matched with defined rules, they are passed to n-gram model. Indika Perera et al [9] proposed a method to classify examination questions into Bloom's cognitive levels using rule based approach. Rules are generated by extracting POS tagging patterns for each level 
and comparison was done by using cosine similarity technique. And also, they used WordNet similarity scores to enhance the classification accuracy by considering the semantic structure of the questions. Wen Chih Chang et al [5] proposed a method to classify examination questions into Bloom's taxonomy cognitive levels by extracting verbs form examination questions and match them with predefined Bloom's taxonomy cognitive levels keywords. For conflicting keywords with multiple levels, they introduced a weighting technique for each keyword.

Most of the recent studies works on question classification are based on a supervised learning method. Supervised learning approaches learn a classifier from a given training set con-sisting of labeled questions. Anbuselvan Sangodiah et al [12] proposed a method to classify question into Bloom's taxonomy cognitive levels using SVM. In his study, he addressed the keyword overlapping problem and he proposed, using powerful feature extraction techniques can solve this issue. Anwar Ali Yahya et al [14] proposed a method to classify examination questions into Bloom's cognitive levels using SVM. They used linear kernel function and SVM-Light software package in their study. Addin Osman et al [3] conducted a case study that compares different machine learning methods such as NB, SVM, logistic regression and decision tree to classify examination questions into Bloom's cognitive levels. Results suggested that SVM and logistic regression models show better accuracy than NB and decision trees. Norazah Yusof et al [15] proposed an Artificial Neural Network (ANN) model with scaled conjugate gradient learning algorithm to classify examination questions into Bloom's cognitive levels.

The combination of two or more classifiers has been used in many fields to improve classification results. Dhuha Abdulhadi Abduljabbar et al [2] proposed a method to classify examination questions into Bloom's taxonomy cognitive levels using the combination of three machine learning classifiers such as SVM, $\mathrm{NB}$ and KNN using majority voting algorithm. They used different feature extraction methods such as chi-square, mutual information, and odd ratio. Ali Danesh et al [6] proposed a method for text classification using three classifier combinations such as NB, KNN and Rocchio. Julio Villena Roman et al [13] proposed a method to categorize texts by integrating machine learning and rule based classifiers. They selected KNN as machine learning classifier because of its simplicity. They used machine learning classifier as based classifier and used rule based classifier to enhance the results.

\section{DESIGN AND METHODOLOGY}

The design contains four main modules named preprocessing module, feature extraction module, classification module and combination module. One of the major contributions of this research is the method used to get the final classification output from four base classifiers outputs by considering WordNet similarity values. Fig. 2 illustrates the overall design architecture of this study including all the main modules and their sub-processes.

The first component of the question classification system is preprocessing module. In order to prepare the ill-formatted input data for the further process, each question has to be normalized using simple preprocessing techniques mentioned. Decomposing natural language questions into features is a focal task of any question classification system, and this is defined as the second module of the system named as feature extraction module. This

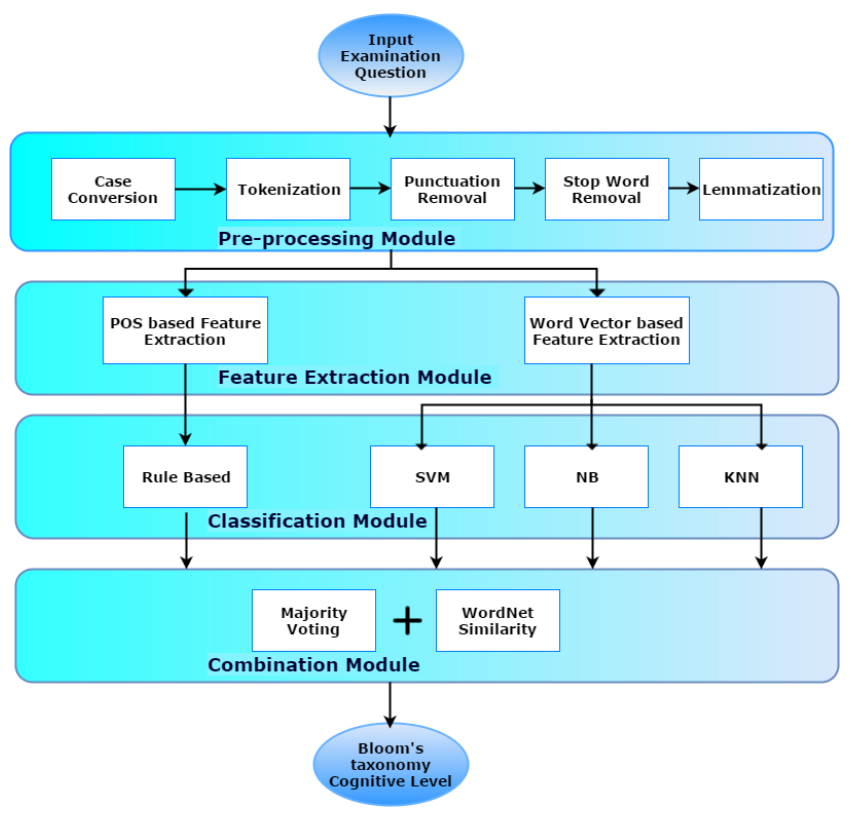

Fig. 2. Overall design architecture for ensemble classifier approach

module is responsible for the extracting set of features from preprocessed question. Feature extraction is achieved by two subroutines, part of speech based feature extraction for rule based classification and word vector based feature extraction for machine learning classifications. The third component of this system is classification modules, which uses feature vectors of examination questions and classify them into Bloom's taxonomy cognitive levels by using four classifiers such as rule based classifier, support vector machine, Naive Bayes and k-nearest neighbor. The final component of this system is combination module which combines results of four base classifiers using the maximum voting algorithm with WordNet similarity values and then outputs the corresponding Bloom's taxonomy cognitive level for given input examination question.

Implementation of the rule based approach was inspired by the research of Indika Perera et al [9]. Python 3.4 is used as the programming language for the implementation of this phase. Implementation of the machine learning approach was inspired by the research of Dhuha Abdulhadi et al [2]. The tool used for machine learning classification is Weka machine learning software.

\section{EVALUATION AND RESULTS}

\subsection{Reference Data Set}

For the evaluation process, this study has selected a dataset which contains first year programming examination questions of University of Colombo School of Computing. This dataset contained hundred programming examination questions and divide this dataset into two parts namely training set and testing set. The training set consists with sixty examination questions and it used for training the classifiers and builds models. Testing set consist of forty examination questions and it used for testing the accuracy of classifiers. 


\subsection{Evaluation Metrics}

For the evaluation, this study has used few measures namely, Precision, Recall, F-measure and accuracy for understanding and measuring relevance and the correctness of the examination question classification into Bloom's Taxonomy cognitive levels. Recall measures the completeness of the quality of the results. Precision reflects the exactness of the quality of the results. F-measure is the harmonic mean of the precision and recall of a test and is used to measure the accuracy.

\subsection{Experiments}

Evaluation of this study was performed on the dataset described in above section. All the experiments of this study can be categorized as three ways,

(1) Experiment I - Individual Classifier Experiment :

Analyze individual classifier results (Rule Based, SVM, KNN, $\mathrm{NB}$ ).

(2) Experiment II - Ensemble Machine Learning Classifier Experiment:

Analyze ensemble machine learning classifier results (SVM, $\mathrm{KNN}, \mathrm{NB}$ ).

(3) Experiment III - Ensemble Rule Based and Machine Learning Classifier Experiment:

Analyze ensemble classifier results (Rule Based + SVM + $\mathrm{KNN}+\mathrm{NB}$ ).

\subsubsection{Experiment I - Individual Classifier Experiment.}

In this section, this study performed four experiments for each four different classifiers and obtained the results.

\subsubsection{Rule Based Classifier.}

Table 1 represents evaluation metrics of rule based classifier for each Bloom's Taxonomy cognitive levels. And here this study observed that evaluate and create classes are successfully classified by this classifier, but for other remaining classes it showed poor results. The overall accuracy of the rule based classifier is $60 \%$.

Table 1. Evaluation metrics for rule based classifier

\begin{tabular}{|c|c|c|c|c|c|c|}
\hline Class & Rem. & Und. & App. & Ana. & Eva. & Cre. \\
\hline Precision & 1 & 0 & 0.5 & 0.29 & 0.45 & 0.73 \\
\hline Recall & 0.71 & 0 & 0.2 & 0.4 & 1 & 1 \\
\hline F-measure & 0.83 & - & 0.29 & 0.34 & 0.62 & 0.84 \\
\hline
\end{tabular}

\subsubsection{Support Vector Machine.}

Table 2 represents evaluation metrics of support vector machine for each Bloom's Taxonomy cognitive levels. And here this study observed this classifier successfully classifies all the cognitive levels with much accuracy and remember level is classified as $100 \%$. The overall accuracy of SVM classifier is $72 \%$.

Table 2. Evaluation metrics for SVM classifier

\begin{tabular}{|c|c|c|c|c|c|c|}
\hline Class & Rem. & Und. & App. & Ana. & Eva. & Cre. \\
\hline Precision & 1 & 0.5 & 0.4 & 1 & 0.66 & 1 \\
\hline Recall & 1 & 0.43 & 0.8 & 0.4 & 0.8 & 0.81 \\
\hline F-measure & 1 & 0.46 & 0.53 & 0.57 & 0.72 & 0.89 \\
\hline
\end{tabular}

\subsubsection{Naive Bayes Classifier.}

Table 3 represents evaluation metrics of naive Bayes classifier for each Bloom's Taxonomy cognitive levels. And here this study observed this classifier successfully classifies all the cognitive levels with much accuracy and remember level is classified as $100 \%$. The overall accuracy of NB classifier is $70 \%$.

Table 3. Evaluation metrics for NB classifier

\begin{tabular}{|c|c|c|c|c|c|c|}
\hline Class & Rem. & Und. & App. & Ana. & Eva. & Cre. \\
\hline Precision & 1 & 0.5 & 0.33 & 1 & 0.66 & 1 \\
\hline Recall & 1 & 0.43 & 0.6 & 0.4 & 0.8 & 0.81 \\
\hline F-measure & 1 & 0.46 & 0.42 & 0.57 & 0.72 & 0.89 \\
\hline
\end{tabular}

\subsubsection{K-nearest Neighbor Classifier.}

Table 4 represents evaluation metrics of k-nearest neighbor classifier for each Bloom's Taxonomy cognitive levels. And here this study observed this classifier successfully classifies all the cognitive levels with much accuracy and remember level is classified as $100 \%$. The overall accuracy of KNN classifier is $72 \%$.

Table 4. Evaluation metrics for KNN classifier

\begin{tabular}{|c|c|c|c|c|c|c|}
\hline Class & Rem. & Und. & App. & Ana. & Eva. & Cre. \\
\hline Precision & 1 & 0.5 & 0.6 & 1 & 0.8 & 1 \\
\hline Recall & 1 & 1 & 0.6 & 0.4 & 0.8 & 0.54 \\
\hline F-measure & 1 & 0.66 & 0.6 & 0.57 & 0.8 & 0.70 \\
\hline
\end{tabular}

\subsubsection{Experiment II - Ensemble Machine Learning Classifier Experiment.}

In this section performed an experiment for combining the results of three machine learning classifiers with majority voting and WordNet similarity algorithm. This experiment was done primarily to compare the results which were done by previous study [2].

Table 5 represents evaluation metrics of ensemble machine learning classifier for each Bloom's Taxonomy cognitive levels. And here this study observed this classifier successfully classifies all the cognitive levels with much accuracy and remember level is classified as $87 \%$. The overall accuracy of ensemble machine learning classifier is $75 \%$.

Table 5. Evaluation metrics for ensemble machine learning classifier

\begin{tabular}{|c|c|c|c|c|c|c|}
\hline Class & Rem. & Und. & App. & Ana. & Eva. & Cre. \\
\hline Precision & 0.77 & 0.5 & 0.6 & 1 & 0.8 & 1 \\
\hline Recall & 1 & 0.71 & 0.6 & 0.4 & 0.8 & 0.54 \\
\hline F-measure & 0.87 & 0.58 & 0.5 & 0.57 & 0.8 & 0.66 \\
\hline
\end{tabular}

\subsubsection{Experiment III - Ensemble Rule Based and Machine Learning Classifier Experiment.}

In this section performed an experiment for combining the results of four classifiers with majority voting and considering WordNet similarity values. This is the real approach proposed in this study Table 6 represents evaluation metrics of ensemble classifier for each Bloom's Taxonomy cognitive levels. And here this study observed this classifier successfully classifies all the cognitive levels 
with much accuracy and remember level is classified as $100 \%$. The overall accuracy of ensemble classifier is $82 \%$.

Table 6. Evaluation metrics for ensemble classifier

\begin{tabular}{|c|c|c|c|c|c|c|}
\hline Class & Rem. & Und. & App. & Ana. & Eva. & Cre. \\
\hline Precision & 1 & 0.6 & 0.6 & 1 & 1 & 0.92 \\
\hline Recall & 1 & 0.86 & 0.6 & 0.4 & 0.8 & 1 \\
\hline F-measure & 1 & 0.71 & 0.6 & 0.57 & 0.88 & 0.95 \\
\hline
\end{tabular}

\subsection{Overall Results Analysis}

According to above results, this study observed the proposed ensemble approach had achieved a considerable value about precision. This means, the exactness of the proposed approach. A higher precision means, less false positives, in other words, the proposed approach has been able to identify the features correctly which has resulted in reducing the classified target features of the examination questions into incorrect cognitive levels.

And also, the proposed ensemble approach has achieved a considerable value about recall. Recall measures the completeness or sensitivity of the classifier. Table 7 shows F-measure values obtained for each of the experiments. Using this table this study observed that for the ensemble classifier approach all the f-measure values are maximized for each Bloom's Taxonomy cognitive level.

Table 7. F-measure values for each experiment

\begin{tabular}{|c|c|c|c|c|c|c|}
\hline- & Rem & Und & App & Ana & Eva & Cre \\
\hline Rule Based & 0.83 & - & 0.29 & 0.34 & 0.62 & 0.84 \\
\hline SVM & 1 & 0.46 & 0.53 & 0.57 & 0.72 & 0.89 \\
\hline KNN & 1 & 0.66 & 0.6 & 0.57 & 0.8 & 0.70 \\
\hline NB & 1 & 0.46 & 0.42 & 0.57 & 0.72 & 0.89 \\
\hline Com.M.L & 0.87 & 0.58 & 0.5 & 0.57 & 0.8 & 0.66 \\
\hline Ensemble & 1 & 0.71 & 0.6 & 0.57 & 0.88 & 0.95 \\
\hline
\end{tabular}

Fig. 3 shows F- measure results as a histogram for all experiments conducted in this study.

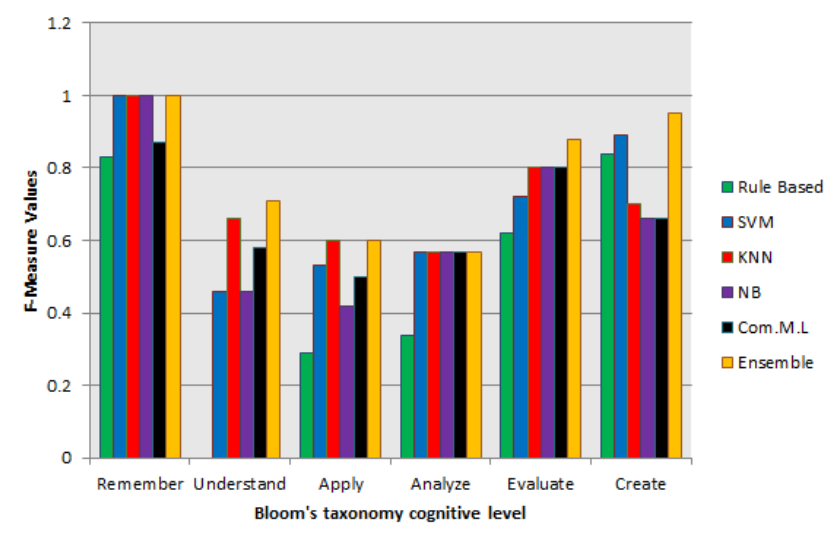

Fig. 3. Histogram for f-measure values of experiments

And also all of the above-mentioned experiments proved that the hypothesis used in this study which is classifier ensembles are based on the reasoning that strengths and weaknesses of various classifiers can compensate each others correct. Because the overall accuracy value for the ensemble classifier is greater than the accuracy value of each single classifier. And also it is greater than the accuracy value of ensemble machine learning classifier. So this study observed that integrating rule based classifier with machine learning classifiers improved the overall accuracy value of the ensemble classifier. Table 8 shows the overall accuracy values for each experiment.

Table 8. Overall accuracy for each experiment

\begin{tabular}{|c|c|c|c|c|c|c|}
\hline Classifier & Ru. Ba & SVM & KNN & NB & Com. M.L & Ens. \\
\hline Accuracy & $60 \%$ & $72.5 \%$ & $72.5 \%$ & $70 \%$ & $75 \%$ & $82.5 \%$ \\
\hline
\end{tabular}

So according to all above results can conclude that the proposed ensemble classifier approach of this study is more accurately classify examination questions into Bloom's Taxonomy cognitive levels than individual classifiers and ensemble machine learning classifier.

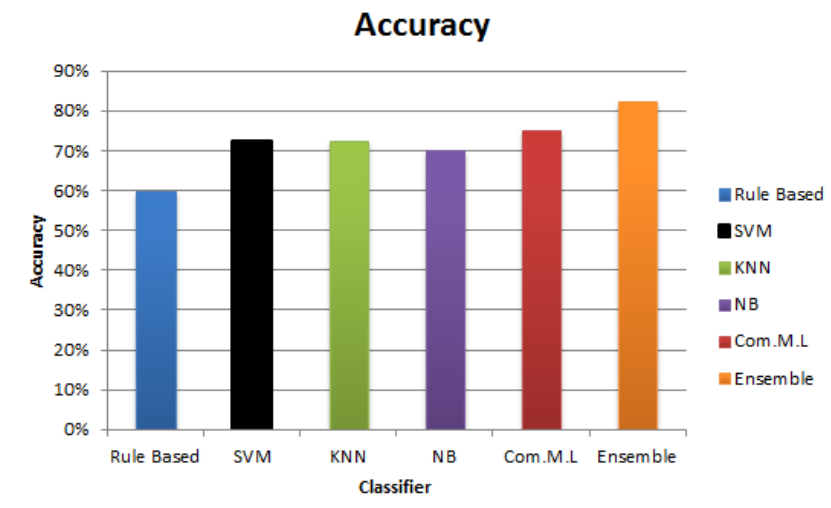

Fig. 4. Histogram for accuracy values of experiments

\section{CONCLUSION AND FUTURE WORKS}

This study is aimed to develop an automated technique for classify examination questions into Bloom's taxonomy cognitive levels. So, this study proposed a method to ensemble the four different classifiers such as rule based, support vector machines, Naive Bayes and k-nearest neighbor to obtain higher accuracy for the classification. This paper has explained all the different stages of development, starting with the construction of the dataset, designing and implementing proposed methodology and evaluation of the study. In order to combine the four different classifier results, this study used majority voting technique with WordNet similarity values to eliminate ambiguous Bloom's Taxonomy cognitive levels. Various tools and techniques have been used for extracting features from examination questions.

The main difference of proposed solution with available research work related to this is an inclusion of semantic similarity using WordNet when combining classifier results to obtain the corresponding Bloom's taxonomy cognitive level for examination questions. It has been observed that semantic feature consideration play a significant role in achieving a decent accuracy in classifying examination questions. So, going beyond mapping exact words with predefined keywords and providing the ability to detect features in an implicit way, based on semantic similarity is explored 
in this study.

In the evaluation phase, this study observed the used hypothesis for this study is true because for the ensemble classifier approach this study derived overall $82 \%$ accuracy and it is greater than the accuracy values of each individual classifier and ensemble machine learning classifier. Since the results basically depend on the dataset chosen in this study, if can add more data to the dataset, this study hope to gain more accuracy for the classification.

The current approach is capable of examination question classification in programming subject domain. Making this study compatible with subject domains other than programming, it needs to add more examination questions to the dataset. A possible future implementation is to identify and extract features in images and paragraphs which are not considered in this study and use those examination questions also for the classification.

The next stage of this study will focus on experimenting with a large size of the dataset in order to obtain more conclusive results. And also, using different feature extraction methods for extracting features from examination questions are also encouraged.

\section{ACKNOWLEDGMENT}

I would like to show my gratitude to Dr.Noel Fernando and Mr.Viraj Welgama for sharing their pearls of wisdom with me during the this research, and I would also like to thank all the lecturers of University of Colombo School of Computing, Sri Lanka for their advises and enormous support.

\section{REFERENCES}

[1] Syahidah Sufi Haris and Nazlia Omar . Determining Cognitive Category of Programming Question with Rule-based Approach. International Journal of Information Processing and Management, 4(3):86-95, 2013.

[2] Dhuha Abdulhadi Abduljabbar and Nazlia Omar. Exam questions classification based on Bloom's taxonomy cognitive level using classifiers combination. Journal of Theoretical and Applied Information Technology, 78(3):447-455, 2015.

[3] Anwar Ali Yahaya Addin Osman. CLASSIFICATIONS of EXAM QUESTIONS USING LINGUISTICALLY- MOTIVATED FEATURES : A CASE STUDY BASED on BLOOM ' S TAXONOMY Research Questions Research Aim. In The Sixth International Arab Conference on Quality Assurance in Higher Education, volume 2016, Saudi Arabia, 2016.

[4] Ibtihal R. Assaly and Oqlah M. Smadi. Using bloom's taxonomy to evaluate the cognitive levels of master class textbook's questions. English Language Teaching, 8(5):100-110, 2015.

[5] Wen Chih Chang and Ming Shun Chung. Automatic applying Bloom's taxonomy to classify and analysis the cognition level of english question items. 2009 Joint Conferences on Pervasive Computing, JCPC 2009, pages 727-733, 2009.

[6] Ali Danesh, Behzad Moshiri, and Omid Fatemi. Improve text classification accuracy based on classifier fusion methods. 2007 10th International Conference on Information Fusion, pages $1-6,2007$.

[7] Eman Ghanem Nayef, Nik Rosila, Nik Yaacob, and Hairul Nizam Ismail. Taxonomies of Educational Objective Domain. International Journal of Academic Research in Business and Social Sciences, 3(9):2222-6990, 2013.
[8] Syahidah Sufi Haris and Nazlia Omar. Bloom's taxonomy question categorization using rules and $\mathrm{N}$-gram approach. Journal of Theoretical and Applied Information Technology, 76(3):401-407, 2015

[9] K. Jayakodi, M. Bandara, I. Perera, and D. Meedeniya. WordNet and cosine similarity based classifier of exam questions using bloom's taxonomy. International Journal of Emerging Technologies in Learning, 11(4):142-149, 2016.

[10] L Moreira-Matias and J Mendes-Moreira. Text categorization using an ensemble classifier based on a mean co-association matrix. Technical report, 2012.

[11] Lior Rokach. Ensemble-based classifiers. Technical Report November 2009, 2010.

[12] Anbuselvan Sangodiah, Rohiza Ahmad, Wan Fatimah, and Wan Ahmad. A Review in Feature Extraction Approach in Question Classification Using Support Vector Machine. 2014 IEEE International Conference on Control System, Computing and Engineering, (November):536-541, 2014.

[13] Julio Villena Román, Sonia Collada Pérez, Sara Lana Serrano, and José Carlos González Cristóbal. Hybrid Approach Combining Machine Learning and a Rule-Based Expert System for Text Categorization. In Proceedings of the Twenty-Fourth International Florida Artificial Intelligence Research Society Conference - Twenty-Fourth International Florida Artificial Intelligence Research Society Conference - 18/05/2011 - 20/05/2011 - Palm Beach, Florida, EEUU, pages 323-328, 2011.

[14] AA Yahya and A Osman. Automatic classification of questions into Bloom's cognitive levels using support vector machines. In The International Arab Conference on ..., number December 2011, 2011.

[15] Norazah Yusof and Chai Jing Hui. Determination of Bloom ' s Cognitive Level of Question Items using Artificial Neural Network. In 10th International Conference on Intelligent Systems Design and Applications (ISDA), pages 866-870, 2010. 